\title{
Protecting the lungs but hurting the kidneys: causal inference study for the risk of ventilation-induced kidney injury in ARDS
}

\author{
Haopu Yang ${ }^{1,2,3}$, Panayiotis V. Benos ${ }^{2}$, Georgios D. Kitsios ${ }^{3,4}$ \\ ${ }^{1}$ School of Medicine, Tsinghua University, Beijing, China; ${ }^{2}$ Department of Computational and Systems Biology, University of Pittsburgh, Pittsburgh, \\ PA, USA; ${ }^{3}$ Center for Medicine and the Microbiome, University of Pittsburgh, Pittsburgh, PA, USA; ${ }^{4}$ Division of Pulmonary, Allergy and Critical \\ Care Medicine, Department of Medicine, University of Pittsburgh School of Medicine and University of Pittsburgh Medical Center, Pittsburgh, PA, \\ USA \\ Correspondence to: Georgios Kitsios, MD, PhD. Assistant Professor of Medicine, Division of Pulmonary, Allergy and Critical Care Medicine, \\ University of Pittsburgh, UPMC Montefiore Hospital, NW 628, 3459 Fifth Avenue, Pittsburgh, PA 15213, USA. Email: kitsiosg@upmc.edu. \\ Provenance and Peer Review: This is an invited article commissioned by the Editorial Office, Annals of Translational Medicine. The article did not \\ undergo external peer review. \\ Comment on: Leite TT, Gomes CAM, Valdivia JMC, et al. Respiratory parameters and acute kidney injury in acute respiratory distress syndrome: a \\ causal inference study. Ann Transl Med 2019;7:742.
}

Submitted Feb 29, 2020. Accepted for publication Apr 28, 2020.

doi: $10.21037 /$ atm-20-2050

View this article at: http://dx.doi.org/10.21037/atm-20-2050

The acute respiratory distress syndrome (ARDS) is the most serious form of acute respiratory failure in the intensive care unit (ICU), defined by hypoxemia and bilateral opacities on chest radiographic imaging (1). ARDS can represent the end-result of many different insults—-such as sepsis, pneumonia, aspiration or pancreatitis-leading to injury of the alveolar epithelial/endothelial interface with resultant permeability edema (2). Epidemiologically, ARDS accounts for up $10 \%$ of all ICU admissions and can be diagnosed in up to $23 \%$ of mechanically-ventilated patients (2). Severe ARDS carries $30-40 \%$ mortality risk, whereas ARDS survivors often suffer from chronic critical illness and longterm neurocognitive deficits (3). Despite advancements in understanding ARDS pathogenesis, no pharmacologic therapy for ARDS has shown efficacy in clinical trials. Thus, invasive mechanical ventilation (IMV) remains the mainstay of ARDS management, with the goals of supporting gas exchange, allowing the respiratory system to "rest", and providing time to treat the underlying pathology.

Decades of research have shown that despite its beneficial effects in gas exchange, IMV can also precipitate and perpetuate severe lung injury. Ventilation-induced lung injury (VILI) encompasses a spectrum of mechanisms that relate to the delivery of positive-pressure ventilation in lungs with low compliance and heterogeneously distributed pathology (i.e., worse edema in dorsal lung units and small amounts of normally aerated lung ventrally). Over-distention of the normally aerated alveoli due to inappropriately high tidal volumes results in stretch injury (volutrauma), with further risks of alveolar rupture and air leak (e.g., pneumothorax) (4). Conversely, ventilation at inappropriately low volumes leads to cyclic alveolar opening and collapse, with ensuing repetitive epithelial injury from shear forces (atelectrauma). Such mechanisms converge into the release of inflammatory mediators from the injured lung (e.g., interleukin-6 and tumor necrosis factor-alpha), which can leak in the systemic circulation due to the increased alveolar/capillary permeability and cause distant end-organ damage (biotrauma) (5). To mitigate VILI, multiple lung protective ventilatory strategies have been examined, yet only two elements have consistently proven to be important: low tidal volume ventilation (typically prescribed at $6 \mathrm{~mL} / \mathrm{kg}$ of ideal body weight) to prevent volutrauma (6) and the application of positive end-expiratory pressure (PEEP) to prevent atelectrauma (7). Nonetheless, delivering an optimal PEEP individualized to patient-specific respiratory physiology remains an issue of ongoing debate (7).

While lung protective ventilation remains the central tenet of ARDS management, the extrapulmonary consequences of IMV cannot be overlooked. Patients with ARDS often develop cardiovascular, renal and neurologic dysfunctions, and then succumb to consequences of 
A

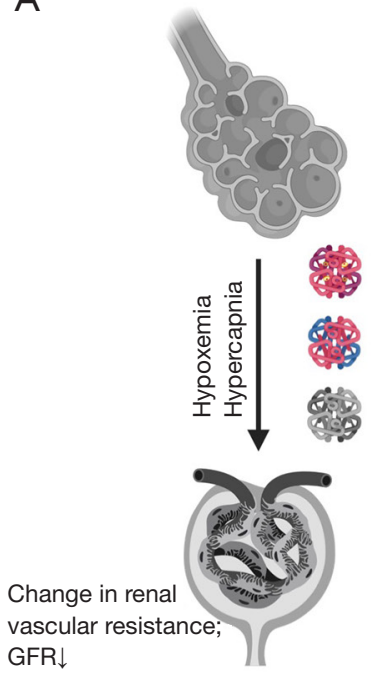

B

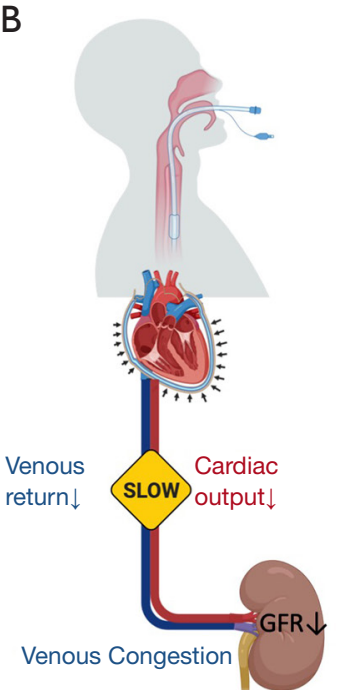

C

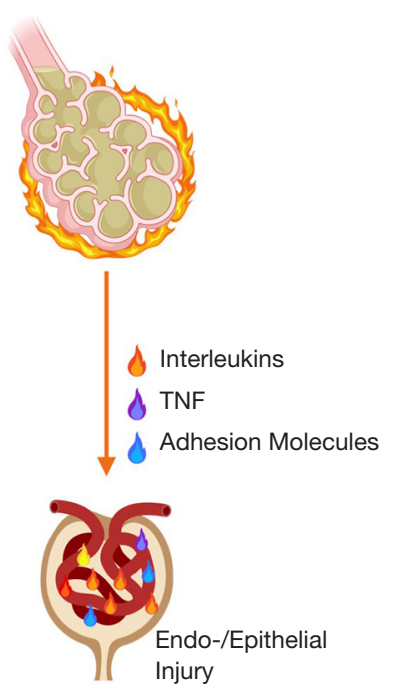

Figure 1 Mechanisms of plausible mechanisms of ventilator-induced kidney injury. (A) Gas exchange disturbances, which typically include hypoxemia and hypercapnia, lower renal vascular resistance and lead to a drop in glomerular filtration rate (GFR); (B) hemodynamics changes during invasive mechanical ventilation (IMV) can cause an increase in intrathoracic and right atrial pressures and thus a decrease in cardiac output and venous return, consequently lowering GFR; (C) inflammatory mediators from the lungs trigger both endothelial and epithelial injury in glomeruli and renal tubules (not shown). TNF, tumor necrosis factor.

multiple organ failure syndrome rather than to refractory hypoxemia. Acute kidney injury (AKI) is the most frequent extrapulmonary organ damage in ARDS and affects up to $50 \%$ of patients, doubling their mortality risk compared to patients without AKI $(8,9)$. Initiation of IMV is associated with a threefold increase in the odds of AKI, a finding consistent among populations of critically-ill patients, regardless of ARDS diagnosis (10). Such epidemiologic observations fueled interest in understanding the mechanisms involved in the cross-talk between the lungs and the kidneys that can lead to ventilator-induced kidney injury (VIKI) (11).

Data from clinical studies and physiologic experiments for plausible lung-kidney interactions during IMV can be synthesized into three main mechanisms (Figure 1). First, gas exchange disturbances from the primary lung pathology can exert direct effects to the kidneys. The hallmark hypoxemia of ARDS and permissive hypercapnia of lungprotective ventilation can alter renal vascular resistance and perfusion pressures, resulting in a drop of glomerular filtration rates (GFR), ischemic acute tubular necrosis and finally, clinical AKI (Figure 1A) $(11,12)$. Second, the kidneys are directly exposed to the hemodynamic effects of IMV on the systemic circulation. Positive pressure ventilation increases the intrathoracic and right atrial pressures, impairing right ventricular venous return and leading to renal venous congestion. Apart from the negative effects on right ventricular preload, intrathoracic pressures also raise the right ventricular afterload, with a net reduction of cardiac output. As recipients of a diminished cardiac output, the kidneys are exposed to the double hit of lower renal blood flow coupled with venous congestion, ultimately dropping renal perfusion pressures and GFR (Figure 1B). Finally, renal glomeruli and tubules are also exposed to the inflammatory mediators released from the "leaky" injured lungs of ARDS (biotrauma), which can further disturb intra-renal hemodynamics, vascular tone and endothelial/epithelial integrity (Figure 1C) (8). The relative contributions of these purported mechanisms in generation of VIKI have not been clarified, yet these are important for the design and implementation of kidney protective ventilatory strategies.

In the last issue of the Annals of Translational Medicine, Leite et al. addressed the important question of which parameters of mechanical ventilation parameters directly influence the risk of VIKI, by doing causal inference analyses in an observational cohort study (13). Starting with a large, open-access database (Multiparameter Intelligent Monitoring 
in Intensive Care-MIMIC-III), the authors performed a careful selection of adult patients with ARDS who received IMV for $>48$ hrs with volume-control type of mechanical breaths. In doing so, the authors attempted to minimize heterogeneity in their dataset ( $n=1,142$ patients) in order to then delineate the effects of readily available respiratory parameters on risk of VIKI, which was defined by established criteria of creatinine and urine output metrics (14).

The mechanisms of lung-kidney cross-talk during IMV are intertwined and can translate into a dataset of multiple correlated and mathematically-derived variables. Therefore, the authors used a causal modeling approach with directed acyclic graphs (DAGs) to ascertain which of the statistical associations may represent true causal risk factors for VIKI. DAGs represent a method of increasing popularity in causal inference studies, due to their intuitive visual representation of data and interpretability of results. In contrast to studies examining the use of a set of variables to predict an outcome of interest (i.e., predictive modeling), causal inference studies aim to estimate the direct "causal" effect of an exposure on an outcome, so that causal hypotheses can be generated to guide the design of clinical trials. By definition, a DAG consists of a set of vertices (variables) and a set of (directed) edges, representing conditional dependencies between variables. An edge between $\mathrm{A} \rightarrow \mathrm{B}$ implies that a change in A will "cause" a change in B, independently of all other variables or subsets of in the dataset (15). Using a graph to depict a model enables easy and succinct display of substantive assumptions collectively, simplifies the calculation of the joint probabilistic density function and also provides an intuitive visualization of causal relationship network among variables of interest. Nevertheless, DAGs have certain limitations including the assumption of linearity of interactions and potentially overlooking unobserved confounder variables, although progress has been made in these fronts as well (16). Graphical models can be built by incorporating prior knowledge; or can be done as probabilistic graphical model (PGM) learning directly from observational data, through searching the space of potential graphs to maximizing an objective function (Bayesian Information Criterion, graph stability, etc.); or a combination of two approaches (17). Well-encapsulated tools like $\mathrm{R}$ packages of bnlearn and pcalg are used extensively when datasets contain a single type of variables, either continuous or discrete. As for causal discovery and PGMs, the widely acknowledged causal discovery software TETRAD now can learn graphs over mixed variable types (continuous and discrete data in the same dataset). The tool of graphical modeling is useful in biomedical research when modeling multi-scale data such as identifying genetic variants related to disease onset or response to drugs (18), or building predictive models for disease progression $(19,20)$. In this case of VIKI for example, we are not just interested in whether levels of PEEP would help evaluate risk of VIKI adjusted for all other variables, but also whether high PEEP levels can actually cause VIKI so that then we could titrate PEEP appropriately to reduce the risk of VIKI. For that purpose, building a graphical model representing presumptions collectively would be helpful for analysis of causality.

In the study by Leite et al., the authors constructed a DAG by linking several IMV parameters (such as timeweighted PEEP, driving pressure, plateau pressure, tidal volume), gas exchange metrics $\left(\mathrm{PaO}_{2}, \mathrm{PaCO}_{2}\right.$ and $\mathrm{PaO}_{2} /$ $\mathrm{FiO}_{2}$ ratio), as well as an overarching average respiratory system compliance $\left(\mathrm{C}_{\mathrm{rs}}\right)$ with VIKI, while controlling for other clinical variables considered as confounders (e.g., age, gender, fluid balance, drugs etc.). Following a series of main and sensitivity analyses, two variables were consistently shown to have causal effects with VIKI: $\mathrm{C}_{\mathrm{rs}}$ and PEEP. Each $5-\mathrm{mL} / \mathrm{cmH}_{2} \mathrm{O}$ increase in $\mathrm{C}_{\mathrm{rs}}$ reduced the odds of severe AKI by $10 \%$, whereas each $1-\mathrm{cmH}_{2} \mathrm{O}$ increase in PEEP increased the odds of severe AKI by $5 \%$.

The direct causal relationship of the average compliance of the entire respiratory system $\left(\mathrm{C}_{\mathrm{rs}}\right)$ with VIKI is physiologically intuitive. This summary compliance (defined as change of volume over change of pressure with each mechanical breath, and thus measured as delivered tidal volume divided by the difference between the measured plateau pressure and PEEP) is a crude, global reflection of combined lung and chest wall mechanics. Low $\mathrm{C}_{\mathrm{rs}}$ can thus represent the "stiff" and "wet" lungs of ARDS, as well as a restricted thoracic cavity, from obesity, effusions or increased intra-abdominal pressures. The authors reasonably asserted that the effects of $\mathrm{C}_{\mathrm{rs}}$ on VIKI could be, at least partially, mediated by some of the measured respiratory and gas exchange variables. However, in mediation analyses, only PEEP was found to have a statistically significant (but minimal in size) effect on the $\mathrm{C}_{\mathrm{rs}}$-VIKI association. Thus, the demonstration of a causal effect for diminished $\mathrm{C}_{\mathrm{rs}}$ underlines the complex cross-talk between lungs and kidneys, in that a global metric of respiratory dysfunction is linked to kidney dysfunction. However, this finding is not clearly actionable. Most evidence-based interventions in ARDS effectively aim to improve some of the $\mathrm{C}_{\mathrm{rs}}$ components [e.g., lung protective ventilation to avoid 


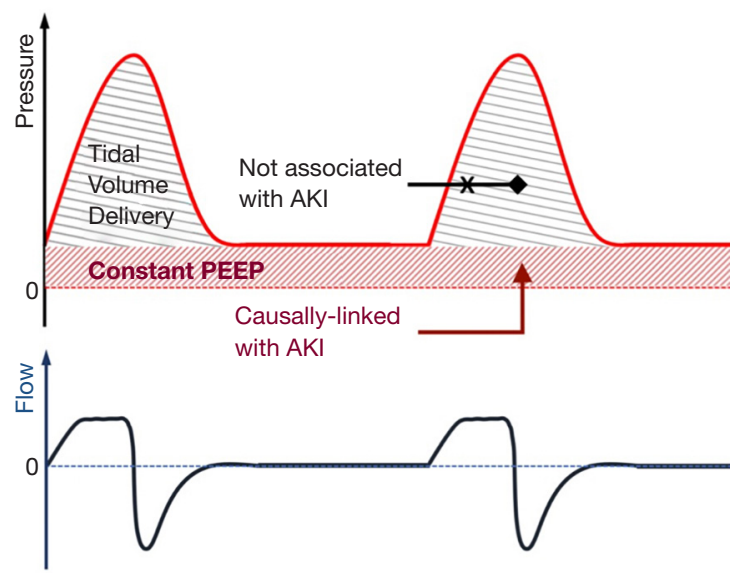

Figure 2 Positive end-expiratory pressure (PEEP) during mechanical ventilation is causally-linked to acute kidney injury (AKI). The study by Leite et al. showed that constantly applied PEEP was causally-linked to AKI while the cyclic rises in pulmonary pressures from tidal volume delivery with each mechanical breath was not.

VILI, conservative fluid management to reduce lung edema and prone positioning to recruit dorsal alveolar units (2)], therefore no clear target for further mitigating VIKI risk emerged from the causal diagram analysis.

On the other hand, the important causal association between PEEP and VIKI in the analysis by Leite et al. may offer opportunities for kidney protective adjustments of ventilatory settings. The near-linear relationship between rising PEEP levels and severe AKI, independent of plateau pressures, suggests that perhaps it is the continuously distending and not the cyclically applied pressure that may hurt the kidneys (i.e., the constant PEEP instead of the intermittent peak/plateau pressures from the delivered tidal volume, Figure 2). The most biologically plausible explanation for the injurious effects of PEEP is through altering kidney hemodynamics (Figure 1B). Nonetheless, the authors were not able to demonstrate any significant mediation of available hemodynamics parameters (central venous or mean pulmonary arterial pressure) between high PEEP and VIKI. Whether this mediation analysis in a subset of the cohort failed to detect the hemodynamic consequences of PEEP due to small sample size or whether PEEP exerts its adverse effects on unmeasured hemodynamic (e.g., intra-abdominal pressure and renal venous congestion) or other parameters remains unknown. Converging evidence suggests that overzealous PEEP application to accomplish maximal alveolar recruitment (“open lung approach") is hemodynamically detrimental and results in worse clinical outcomes $(21,22)$. Ongoing research is trying to identify which patients may have a beneficial response to PEEP up-titration, defined either by improvements in gas exchange, lung mechanics or imaging markers (23). Emerging data suggest that complex, biological endotypes of ARDS $(24,25)$ may offer improved insights in understanding treatment-effect heterogeneity and targeting our ventilatory (and other) interventions. This framework of predictive enrichment by ARDS biological endotypes may help us better model and understand the effects of IMV on extrapulmonary organ damage.

The importance of delivering patient-centric-rather than lung-centric-care in ARDS is self-evident. The study by Leite $e t$ al. reminds us of the knowledge gaps in trying to accomplish this well-shared goal. Supporting the failing lungs with evidence-based, lung-protective IMV settings may still have deleterious, yet underappreciated effects on extra-pulmonary organ functions, which ultimately, are the key determinants of outcome in ARDS. The statistical hits for causal effects of $\mathrm{C}_{\mathrm{rs}}$ and PEEP on the risk of VIKI are biologically plausible, and should be considered as hypothesis-generating for further prospective study; a validation within a larger, multi-center cohort as well as considering a PGM for causal discovery would certainly make such assertions more robust. Assembling the causality chain of VIKI and other organ injury in ARDS is necessary for refining IMV approaches from their current lungprotective to a broader, patient-protective paradigm.

\section{Acknowledgments}

Figure 1 was created with Biorender.com.

Funding: Dr. GDK is being funded from NIH, NHLBI K23 HL139987; Dr. PVB is being funded from NIH, U01 HL137159 and R01 HL140963; Mr. HY is a medical student at Tsinghua University School of Medicine and he is currently a visiting scholar at the University of Pittsburgh School of Medicine, supported by the Tsinghua Educational Foundation North America and the Scholarship Fund of the China Scholarship Council.

\section{Footnote}

Conflicts of Interest: All authors have completed the ICMJE uniform disclosure form (available at http://dx.doi. org/10.21037/atm-20-2050). HY reports other from China Scholarship Council, outside the submitted work; GDK 
reports grants from Karius Inc., outside the submitted work. The other author has no conflicts of interest to declare.

Ethical Statement: The authors are accountable for all aspects of the work in ensuring that questions related to the accuracy or integrity of any part of the work are appropriately investigated and resolved.

Open Access Statement: This is an Open Access article distributed in accordance with the Creative Commons Attribution-NonCommercial-NoDerivs 4.0 International License (CC BY-NC-ND 4.0), which permits the noncommercial replication and distribution of the article with the strict proviso that no changes or edits are made and the original work is properly cited (including links to both the formal publication through the relevant DOI and the license). See: https://creativecommons.org/licenses/by-nc-nd/4.0/.

\section{References}

1. ARDS Definition Task Force, Ranieri VM, Rubenfeld GD, et al. Acute respiratory distress syndrome: the Berlin Definition. JAMA 2012;307:2526-33.

2. Thompson BT, Chambers RC, Liu KD. Acute respiratory distress syndrome. N Engl J Med 2017;377:562-72.

3. National Heart, Lung, and Blood Institute PETAL Clinical Trials Network, Moss M, Huang DT, et al. Early neuromuscular blockade in the acute respiratory distress syndrome. N Engl J Med 2019;380:1997-2008.

4. Gajic O, Dara SI, Mendez JL, et al. Ventilator-associated lung injury in patients without acute lung injury at the onset of mechanical ventilation. Crit Care Med 2004;32:1817-24.

5. Slutsky AS, Ranieri VM. Ventilator-induced lung injury. N Engl J Med 2013;369:2126-36.

6. Brower RG, Shanholtz CB, Fessler HE, et al. Prospective, randomized, controlled clinical trial comparing traditional versus reduced tidal volume ventilation in acute respiratory distress syndrome patients. Crit Care Med 1999;27:1492-8.

7. Walkey AJ, Del Sorbo L, Hodgson CL, et al. Higher PEEP versus Lower PEEP Strategies for Patients with Acute Respiratory Distress Syndrome. A Systematic Review and Meta-Analysis. Ann Am Thorac Soc 2017;14:S297-303.

8. Liu KD, Glidden DV, Eisner MD, et al. Predictive and pathogenetic value of plasma biomarkers for acute kidney injury in patients with acute lung injury. Crit Care Med
2007;35:2755-61.

9. Darmon M, Clec'h C, Adrie C, et al. Acute respiratory distress syndrome and risk of AKI among critically ill patients. Clin J Am Soc Nephrol 2014;9:1347-53.

10. van den Akker JPC, Egal M, Groeneveld ABJ. Invasive mechanical ventilation as a risk factor for acute kidney injury in the critically ill: a systematic review and metaanalysis. Crit Care 2013;17:R98.

11. Husain-Syed F, Slutsky AS, Ronco C. Lung-Kidney CrossTalk in the Critically Ill Patient. Am J Respir Crit Care Med 2016;194:402-14.

12. Sharkey RA, Mulloy EM, O’Neill SJ. Acute effects of hypoxaemia, hyperoxaemia and hypercapnia on renal blood flow in normal and renal transplant subjects. Eur Respir J 1998;12:653-7.

13. Leite TT, Gomes CAM, Valdivia JMC, et al. Respiratory parameters and acute kidney injury in acute respiratory distress syndrome: a causal inference study. Ann Transl Med 2019;7:742.

14. KDIGO. KDIGO Clinical Practice Guideline for Acute Kidney Injury. KDIGO Int 2012;8.

15. Sedgewick AJ, Shi I, Donovan RM, et al. Learning mixed graphical models with separate sparsity parameters and stability-based model selection. BMC Bioinformatics 2016;17 Suppl 5:175.

16. Raghu VK, Ramsey JD, Morris A, et al. Comparison of strategies for scalable causal discovery of latent variable models from mixed data. Int J Data Sci Anal 2018;6:33-45.

17. Manatakis DV, Raghu VK, Benos PV. piMGM: incorporating multi-source priors in mixed graphical models for learning disease networks. Bioinformatics 2018;34:i848-56.

18. Abecassis I, Sedgewick AJ, Romkes M, et al. PARP1 rs1805407 Increases Sensitivity to PARP1 Inhibitors in Cancer Cells Suggesting an Improved Therapeutic Strategy. Sci Rep 2019;9:3309.

19. Sedgewick AJ, Buschur K, Shi I, et al. Mixed graphical models for integrative causal analysis with application to chronic lung disease diagnosis and prognosis. Bioinformatics 2019;35:1204-12.

20. Kitsios GD, Fitch A, Manatakis DV, et al. Respiratory microbiome profiling for etiologic diagnosis of pneumonia in mechanically ventilated patients. Front Microbiol 2018;9:1413.

21. Hodgson CL, Cooper DJ, Arabi Y, et al. Maximal recruitment open lung ventilation in acute respiratory distress syndrome: the PHARLAP trial. Am J Respir Crit Care Med 2020;201:A7310. 
22. Writing Group for the Alveolar Recruitment for Acute Respiratory Distress Syndrome Trial (ART) Investigators, Cavalcanti AB, Suzumura ÉA, et al. Effect of Lung Recruitment and Titrated Positive EndExpiratory Pressure (PEEP) vs Low PEEP on Mortality in Patients With Acute Respiratory Distress Syndrome: A Randomized Clinical Trial. JAMA 2017;318:1335-45.

23. Gattinoni L, Marini JJ, Quintel M. Recruiting the acutely injured lung: how and why? Am J Respir Crit Care Med

Cite this article as: Yang $\mathrm{H}$, Benos PV, Kitsios GD. Protecting the lungs but hurting the kidneys: causal inference study for the risk of ventilation-induced kidney injury in ARDS. Ann Transl Med 2020;8(16):985. doi: 10.21037/atm-20-2050
2020;201:130-2.

24. Calfee CS, Delucchi K, Parsons PE, et al. Subphenotypes in acute respiratory distress syndrome: latent class analysis of data from two randomised controlled trials. Lancet Respir Med 2014;2:611-20.

25. Kitsios GD, Yang L, Manatakis DV, et al. Host-Response Subphenotypes Offer Prognostic Enrichment in Patients With or at Risk for Acute Respiratory Distress Syndrome. Crit Care Med 2019;47:1724-34. 